\title{
The floating point: rounding error in timing devices
}

\author{
David A. Faux* and Janet Godolphin \\ Faculty of Engineering and Physical Sciences, \\ University of Surrey, Guildford, GU2 7XH, UK
}

(Dated: March 8, 2021) 


\section{INTRODUCTION}

A recent article by Faux and Godolphin explored issues of floating-point error in situations relevant to classical dynamics, numerical integration, cellular automata, statistical analysis, and digital timing. ${ }^{1}$ Examples were given that were suitable for discussion and student project work. One of the examples explored the properties of an algorithm, described in an IBM Knowledge Center documen designed to convert a binary field representing the number of counts of a quartz oscillator to integers for digital display. ${ }^{2}$ In Ref. 1 it was demonstrated that the algorithm was vulnerable to rounding error resulting in an incorrect digital display. Investigation associated with this example form the focus of this note.

The timing simulation results presented in Ref. 1 suggested that uncorrected rounding error in stopwatch timer displays could be impactful if used for precision timing, such as for race times or in experimental physics. Here we present and analyse race times obtained from swimming competitions. The data give a clear demonstration of anomalous stopwatch timing patterns, which can only be explained by rounding error. It is also shown that such rounding error can result in a set of times being wrongly ordered. In the context of a sporting event this could lead to the incorrect ranking of athletes and hence the incorrect awarding of race positions. As a spin off of Ref. 1, this note may be of interest to educators, with the results providing a resource for discussion and the approach providing a template for additional student projects.

\section{A STOPWATCH TIMING ANOMALY}

In many sports, including track athletics and swimming, precision timing is essential. Race times are typically measured using digital devices and sent to display to be viewed by the spectators. Given a large enough volume of race data, any systematic rounding error should be identifiable from an anomalous statistical distribution of digits. Race times for track athletes are publicly available but may not be accessible in sufficient numbers for statistically significant conclusions to be drawn from an analysis of the frequency of occurrence of display digits. Swimming is the ideal sport to yield high-volume data because a single three-hour competition typically yields 300-400 race times and these are normally available through the MeetManager application or online. ${ }^{3}$ 
Race times from two consecutive swimming competitions involving a total of 647 stopwatch-timed swims were analysed. ${ }^{4}$ The competitions took place in a six-lane pool. Times were recorded by a timekeeper in each lane, using personal and club stopwatches from a variety of manufacturers. The results for the frequency of the final two displayed digits (the tenth and hundredth of a second) are presented in Fig. 1. Particular pairs of final digits should not be preferred to others. The results appear to be anomalous: there are three digit pairs, namely 00,50 and 75, which together account for more than one eighth of the results; conversely there are eight digit pairs with frequency zero.

A Pearson $\chi^{2}$ goodness-of-fit test was conducted on the set of 647 swim times. Under the null hypothesis that each hundredth was equally likely in each race time, the expected value of the test statistic is 99 and a test statistic exceeding 125 would indicate that the data deviated significantly from the null hypothesis. The realisation of the test statistic is 740 , which exceeds the expected value under the null hypothesis by approximately 45 standard deviations, a statistically impossible outcome if each hundredth is equally likely.

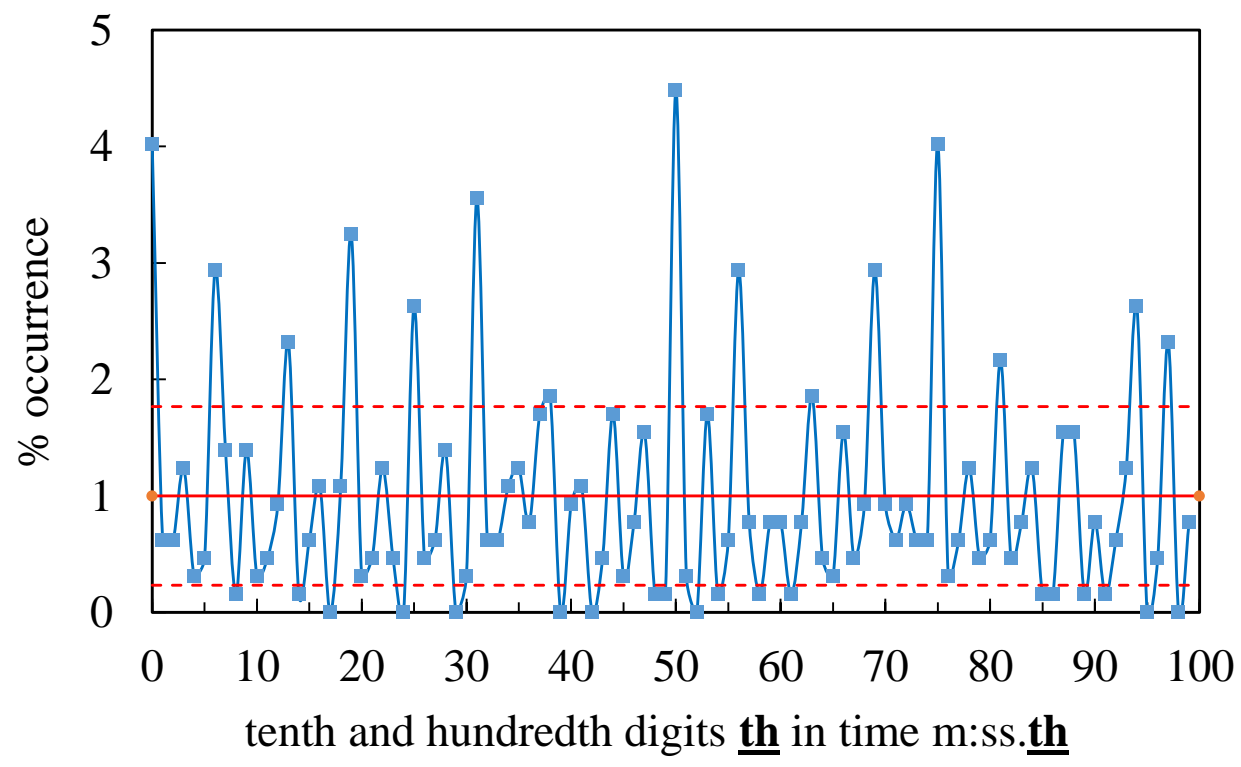

FIG. 1. The $\%$ occurrence of the final two displayed digits of manual race times obtained from two swimming competitions is presented. The expected mean $1 \%$ occurrence and standard deviation of each digit pair are indicated by the red solid and red dashed lines respectively. The observed distribution is statistically impossible if each hundredth is equally likely.

49 Typical swimming race times for distances up to $100 \mathrm{~m}$ range from around $28 \mathrm{~s}$ to $120 \mathrm{~s}$, 
depending on the event and the competitor age. A computer simulation of the process of converting a race time measurement to a digital display time (on a stopwatch in the case of the swimming competitions) was undertaken using the procedure presented in Fig. 6 of Ref. 1 and implemented in a FORTRAN program. ${ }^{5}$ Starting from a binary digit representing (close to) $28 \mathrm{~s}$, all times were processed at single tick intervals to $120 \mathrm{~s}$. This resulted in a set of 3014615 simulated times which were presented in the form sss.th using the standard algorithm. ${ }^{2}$ Prior to going through the display routine each tenth and hundredth digit pair $\underline{\text { th}}$, equal to $00,01 \ldots 99$ in the time format sss.th, had frequency $30146( \pm 1)$. This is entirely consistent with the expectation that, on average, each pair of digits should appear with a probability of $1 \%$. However, after passage through the display routine some tenth and hundredth digit pairs occurred as few as 6873 times in the simulated digital display, whilst others occurred as many as 81606 times. Figure 2 presents the frequencies of digit pairs th after passage through the display routine, expressed as percentages. The oscillations exhibited in Figure 2 are inconsistent with each digit pair occurring with a probability of $1 \%$ and the parallels with the real display data in Fig. 1 are obvious.

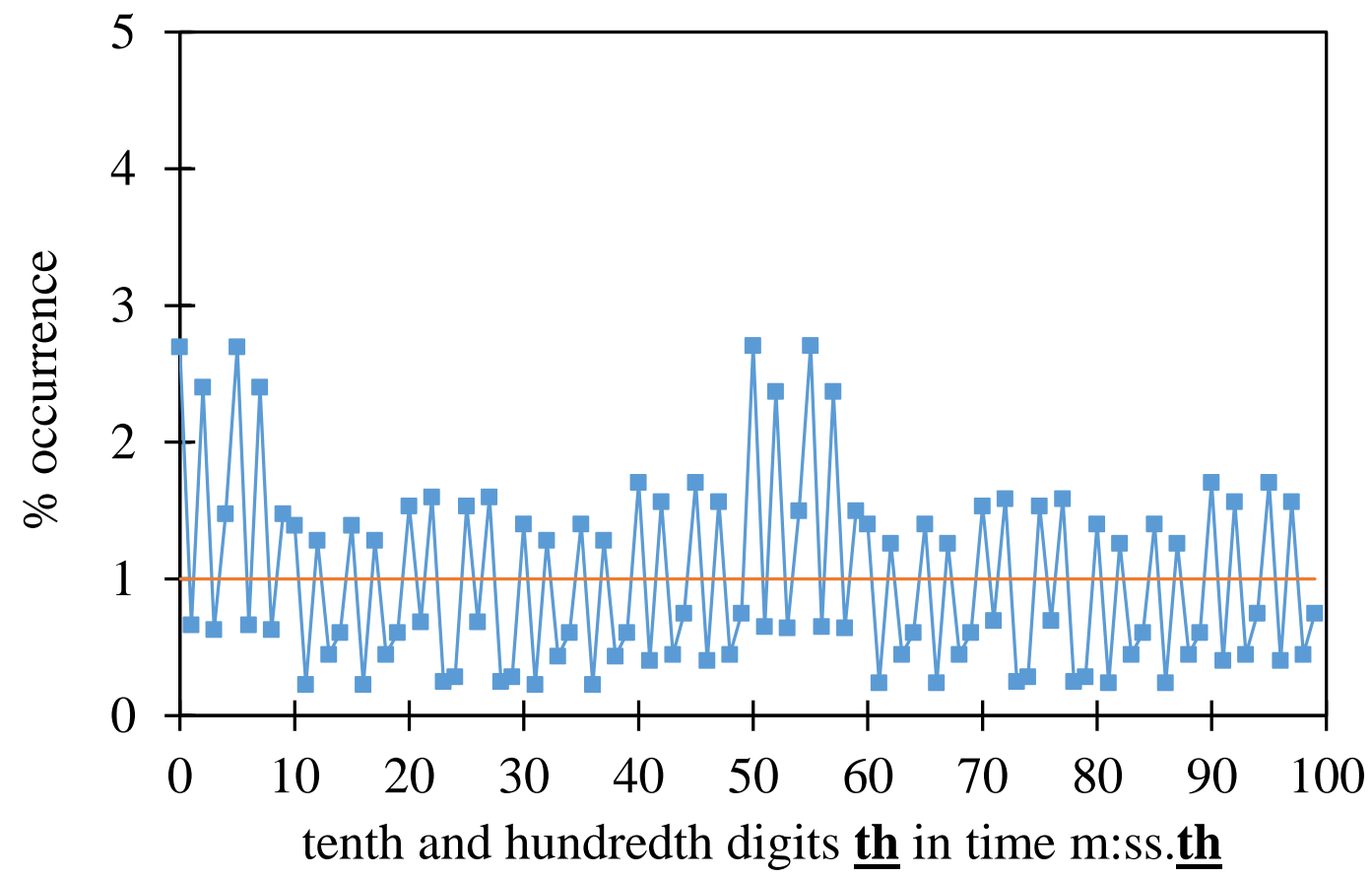

FIG. 2. The frequency of occurrence of each pair of final display digits is presented for the output of a computer simulation which presents race times in stopwatch display format. The expected mean $1 \%$ occurrence is indicated by the orange solid line. The standard deviation is too small to display. 
The distribution of the th digit pairs in Fig. 2 from the simulation of display times is due to uncorrected rounding error. Likewise, rounding errors in stopwatch display software provides the only plausible explanation for the anomalous data in Fig. 1. We have explored other datasets, and undertaken some home tests on the same stopwatches used to produce the data in Fig. 1. All the tests indicated statistically significant departures from the expected mean occurrence of the tenths and hundredths digit pairs and of the hundredth digit, but the data set presented here is the most extreme. There are obvious implications for the accuracy of race results obtained using stopwatches.

\section{CAN ROUNDING ERROR REVERSE THE RANKING OF ATHLETES?}

Any algorithm that converts an integer quartz oscillator count to a floating-point time, and subsequently to integer digits for display, will be subject to rounding error. For the overwhelming majority of time-display applications, this is of no consequence. However, stopwatches are relied on for accurate timing and Fig. 1 provides compelling evidence that they are subject to uncorrected rounding error. Therefore, race times obtained by stopwatches, or results in laboratory physics experiments measured by stopwatches, may be subject to rounding error, which is similar in magnitude to the uncertainty associated with human reaction time. ${ }^{6}$

Consider a sequence of times, in increasing order of duration. From Fig. 1 and Fig. 2 it is unclear whether the process of converting such a set of times to digital display causes some time intervals to be stretched and others to be contracted, whilst preserving the order within the set, or whether the process can corrupt the true order of times. We now demonstrate that rounding errors resulting from converting times to digital display can have the consequence of corrupting the true ordering in a set of times.

TABLE I. The table presents a simulated race result in which Swimmer $A$ wins by $0.01 \mathrm{~s}$ despite having the slower true race time. Rounding errors are responsible for the reversal of rankings.

\begin{tabular}{cccc}
\hline \hline Athlete & $\begin{array}{c}\text { Quartz } \\
\text { Oscillations }\end{array}$ & $\begin{array}{c}\text { True } \\
\text { time (s) }\end{array}$ & $\begin{array}{c}\text { Scoreboard } \\
\text { display }\end{array}$ \\
Swimmer $A$ & 1660659 & 50.6800 & 50.57 \\
Swimmer $B$ & 1657710 & 50.5900 & 50.58 \\
\hline \hline
\end{tabular}


In the history of sporting events there are many instances of very close races. For example, recall what is arguably the most controversial race in swimming history, the $100 \mathrm{~m}$ butterfly final at the 2008 Beijing Olympics. The event was won by Michael Phelps by $0.01 \mathrm{~s}$ in a race that he appeared to have lost to Milorad Cavić. ${ }^{7}$ The result secured Phelps his seventh gold medal. We emphasise that we have no evidence to suggest that timing anomalies occurred in the Phelps Cavić race or even that they exist in electronic timing systems used at high-level sporting competitions, where rounding error may be corrected. The following investigation assumes that electronic timing systems do not correct for rounding error in the display protocol.

Using the same procedure as described in Ref. 1, a simulation was executed for times which are in the neighbourhood of, but not precisely the same as, those of Phelps and Cavić. We label the two closely matched competitors as Swimmer $A$ and Swimmer $B$. In Table 1 we give an example of output that shows Swimmer $A$ as having a display time $0.01 \mathrm{~s}$ faster than that of Swimmer $B$, despite Swimmer $B$ having the faster true race time by the substantial difference of $0.09 \mathrm{~s}$. The true race times place Swimmer $B$ as the faster swimmer, but the digital times position Swimmer $A$ ahead of Swimmer $B$. The display improperly declares Swimmer $A$ as the winner. The simulation demonstrates a mechanism by which the ranking of two athletes correctly determined based on the true race time can be reversed by the process of presenting to the display, if floating-point errors are uncorrected.

* d.faux@surrey.ac.uk

1 David Faux and Janet Godolphin, "Floating-point error: tales of the unexpected," Am. J. Phys. $\mathbf{x x}(2021)$.

2 Converting binary fields to time of day format, IBM Knowledge Center, at https: //www.ibm.com/support/knowledgecenter/en/SSLTBW_2.1.0/com.ibm.zos.v2r1.ieag200/ cnvttime.htm [Accessed: February 2021]

3 "Meet Mobile" is a swim meet application that displays race times by individual, club or competition in almost real time. For information see https://www.active.com/mobile/ meet-mobile-app [Accessed: February 2021].

4 The two competitions were organised by the Hart Swimming Club and held at the Hart Leisure 
117

118

119

120

121

122

Centre, Fleet, Hampshire, UK, in September and October 2017.

5 The computer code is provided as supplementary material.

6 David Faux and Janet Godolphin, "Manual timing in physics experiments: Error and uncertainty", Am. J. Phys. 87 (2), 110-115 (2019).

7 J. Finn, "Timing and imaging evidence in sport: Objectivity, Intervention, and the limits of technology.” J. Sport Soc. Issues, 40, 459-476 (2016). 